\title{
Internet Operators Dominate the Treatment of Food and Drug Rumors--Taking WeChat as an Example
}

\author{
Huimin Yu, Xiaoya Wang, Han Sun, Shuangyan $\mathrm{Yu}^{\mathrm{a}}$ \\ College of economics and management of Jiangxi University of Traditional Chinese Medicine \\ Nanchang 430000, China. \\ a2933788893@qq.com
}

\begin{abstract}
This paper discusses the effective means for the network operators to deal with the online rumor of food and medicine. Analyzing the complaint function of WeChat, WeChat's small assistant of refuting rumors and joining the National Monitoring Center of Public opinion. The effect of WeChat on the treatment of food and drug online rumors is analyzed for finding out the best means of food and drug rumor management. 1. WeChat's complaint function is closely linked with the masses; 2. WeChat's small assistant played the role of leading experts to participate in refuting rumors and popularizing science to the public in a timely manner; 3 . Participating in national health media group food and drug public opinion monitoring center, realizing the effectively cooperation with the national food and drug public opinion supervision. WeChat is more mature in the treatment of drug rumor, which can effectively guide the social co-treatment of food and drug online rumors. The vast number of network operators should learn from their experience and guide the community to participate in the treatment of drug rumors.
\end{abstract}

Keywords: Network operators; WeChat; Food and Drug rumors.

\section{Introduction}

China has entered the Internet age. According to the data of the 41st China Internet Network Development Statistics report, by December 2017, the number of Internet users in China has reached 772 million, and the popularization rate has reached 55.8 percent 1 . With the innovation of Internet mode in China, all kinds of network social platforms emerge as the times require, which brings people great freedom of speech. However, because of the anonymity, virtuality and interactivity of the network environment, it is easier to generate online rumors. With the help of the spread of various network platforms, the spread speed is quickened, the spread scope is wider and the influence will be expanded infinitely, even endangering the normal social order.

Despite the publication of the Supreme people's Court in September 2013, Interpretation by the Supreme people's Procuratorate on several issues applicable to criminal cases such as libel using the information network. "it clarifies the circumstances under which online rumors constitute a crime2. However," without authorization, the law prohibits, A lot of online rumors walk on the edge of the law and have a negative effect on the related things. At the same time, the cause of rumor is often multi-level, such as eyeball game, opportunity marketing, blindly following the trend. Many groups promote the spread of rumors, it is difficult to define the main responsible person. Therefore, in the current situation of insufficient legal basis and cumbersome process of pursuing responsibility, breaking rumors lies in timely refuting rumors, popularizing science and preventing trouble in the early stages.

WeChat, a free app for instant messaging services for smart terminals, was launched by Tencent on January 21, 2011, and is the mobile instant messaging software of Asia's largest user base. WeChat topped the list of Chinese APP users with $96.91 \%$ of active users as of April 2018, according to Media Research. WeChat is a social platform with the largest number of users and the most active users among the existing APP in China. Some studies have proved that the greater the relative importance of rumors, the more involved in the dissemination of groups, will have a greater impact3. Food and drug safety, is the most important in social governance, but also the key points of People's lives. The research on the treatment of food and drug rumors based on WeChat platform, and the analysis of the results obtained, are more useful for the online rumor management. 


\section{Network Operators have Responsibility for Rumor Management}

\subsection{Network Operators have Platform Advantages Over Rumor Governance}

Network operator is the provider of network service and the necessary condition for the wide spread of network rumors. Network is not only anonymous, interactive and virtual, but also has the characteristics of time domain, integration, efficiency, technology and so on. Therefore, the use of network technology, integration of scientific, authoritative information, and timely popularization of science, disinformation, and rumor management will be more efficient and reasonable.

\subsection{Network Operators have Legal Obligations for Rumor Management}

The Law of the people's Republic of China on Cyber Security was formally implemented in China on June 1, 2017. According to Article 49 of the Law on Network Security, network operators shall establish a complaint system for network information security and publish information such as reporting methods, and receive and deal with relevant complaints and reports in a timely manner. The network operator should actively cooperate with the supervision and inspection carried out by the relevant departments according to law 4 . Each network operator has the legal obligation to supervise the public opinion information in their respective network platform in real time and to refute the rumor timely.

\subsection{Internet Operators have a Social Responsibility for Rumor Management}

On the one hand, all kinds of online platforms, as a medium for spreading rumors, are usually owned by a network operator (such as WeChat, a free app launched by Tencent). Enterprises generally refer to legal persons or other social economic organizations that provide goods or services to the market for profit purposes. The spread of rumors attracts more attention for various network platforms, to a certain extent, brings them more potential users and greater prospects for development. Network operation enterprises in the benefit of the same time, should assume the corresponding responsibilities. According to the ten principles of business operation, enterprises should bear the social responsibility of protecting human rights, environmental protection and anti-corruption. For the network environment, the network operation enterprises should bear the social responsibility of maintaining the network environment health and resisting false rumors.

On the other hand, the network rumors spread with the help of network platform. As the "accomplice" of rumor dissemination, each network platform should also bear the corresponding responsibility of rumor management.

\section{WeChat's Three Ways to Deal with Food and Drug Rumors}

As early as 2014, WeChat set up three systems to counter rumors, such as technical interception, manual handling of complaints, and disinformation tools. In recent years, the country pays more and more attention to the work of network security and information, and WeChat is innovating in the management of network rumors. According to the author's own use experience, there are three kinds of methods to deal with food and drug rumors that WeChat has launched at present. Which are complaint function, WeChat disinformation assistant, and China Health Media Group Food and Drug Public opinion Monitoring Center.

\subsection{Complaints Function-reaching Out to the Public}

WeChat users in the discovery of rumors, would use complaints function, provide relevant evidence to report. WeChat will immediately take means to stop the further spread of rumors after WeChat team receives reports from users and verifies that the reports are true. This function is closely related to the masses, users involved in the identification of rumors, promoting social groups to jointly govern rumors. However, the overall level of user culture is uneven, the ability to identify rumors is also different. Especially for food and drug rumors, many users adopt the attitude of "believe in it rather than trust it", or "do not concern yourself, hang up high" attitude. Therefore, the complaint 
function of WeChat has the advantages of connecting the masses and promoting the social cotreatment of online rumors, but also is limited by the lack of users' cognitive level.

\subsection{WeChat Discover Assistant-introduction of Authoritative Institutions}

WeChat disinformation assistant is a Mini Program launched in June 2017. The program is divided into three parts: "rumor recognition", "relevant to me" and "disinformation agency". "rumor recognition" directly shows the recently identified rumors, users can search the search box to find rumors that have already been refuted, "relevant to me" can see the rumors that the user has recently browsed. "disinformation agency" includes a number of rumor-refuting platforms, currently divided into "health class" and "social practice class", users can click on each platform to check out the rumors, can also click on "I want to join" as a rumor-refuting crusader. The author focuses on analyzing the situation of WeChat disinformation assistants in food and drug articles since August 2017 when they were put into normal use until May 2018. Table 1 ( $\mathrm{R}$ for total rumor, FDR for drug rumor, $\mathrm{P}$ for drug rumor). According to the analysis of tables 1 and figure1 and figure 2, since WeChat's rumor-refuting assistant launched, the total number of rumors on WeChat has shown an overall upward trend, but the increase is not obvious, which shows that WeChat's rumor-refuting assistant cannot play a role in containing rumors. However, timely disinformation can prevent the harm caused by the rampant spread of rumors; the proportion of rumors about food and medicine in WeChat has always been kept at 25 to 55, which shows that food and drugs are indeed the top priority in people's lives. "Health" disinformation agencies such as "clove doctors" can scientifically guide people's healthy lives; WeChat disclaimer assistants have introduced "genetic Agriculture Network" and "Hangzhou net Police" to dispel rumors, making the result of refuting rumors more convincing.

Table 1. The Proportion of Food and Drug Rumor among WeChat's Small assistants from August

\begin{tabular}{cccccccccc}
\multicolumn{8}{c}{2017 to May 2018 } \\
\hline & $8.10-9.9$ & $9.10-10.9$ & $10.10-11.9$ & $11.10-12.9$ & $12.10-1.9$ & $1.10-2.9$ & $2.10-3.9$ & $3.10-4.9$ & $4.10-5.9$ \\
\hline $\mathrm{R}$ & 24 & 12 & 7 & 12 & 7 & 23 & 24 & 42 & 28 \\
$\mathrm{FDR}$ & 8 & 3 & 3 & 4 & 3 & 8 & 7 & 23 & 13 \\
$\mathrm{P}$ & $33.33 \%$ & $25.00 \%$ & $42.86 \%$ & $33.33 \%$ & $42.86 \%$ & $34.78 \%$ & $29.17 \%$ & $54.76 \%$ & $46.43 \%$ \\
\hline
\end{tabular}

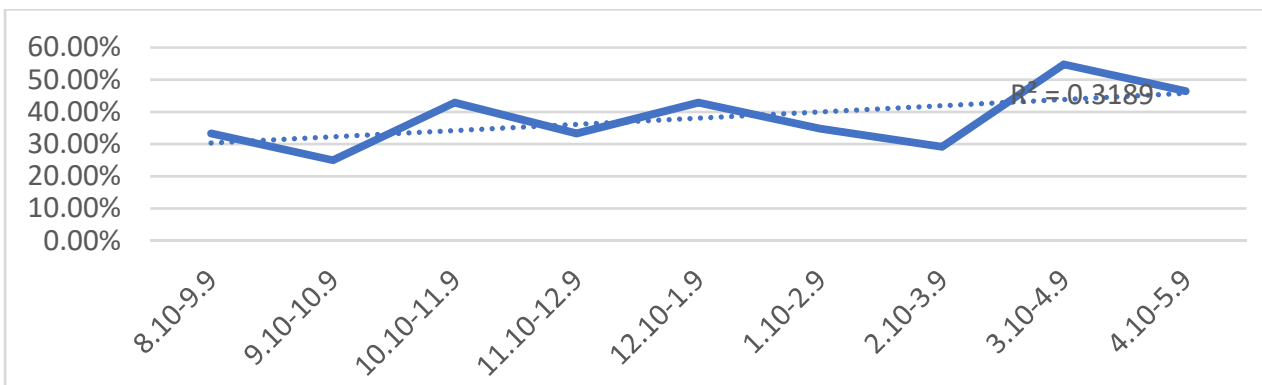

Fig 1. WeChat small assistant refuting rumors from August 2017 to May 2018

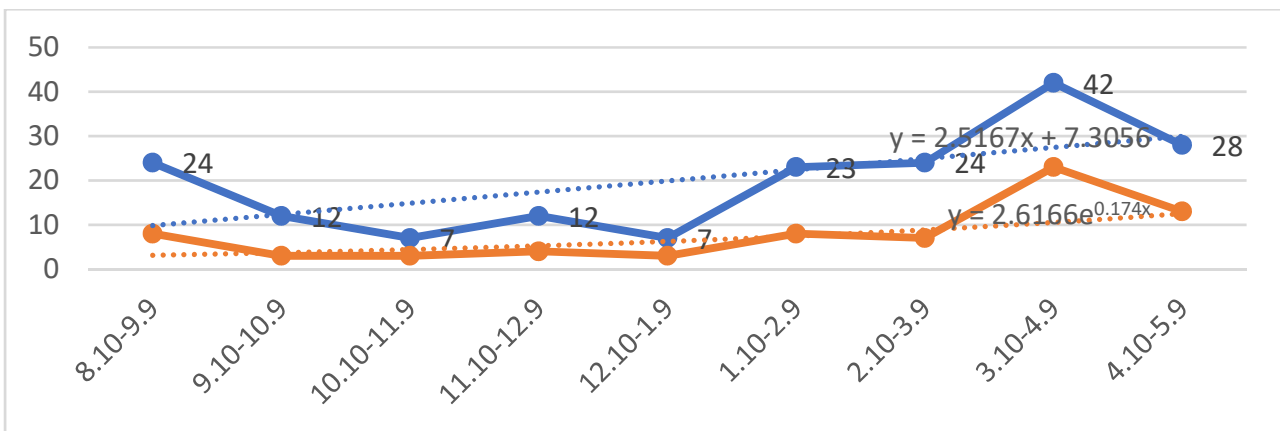

Fig 2. the proportion of rumors in WeChat from August 2017 to May 2018 


\subsection{Join the Food and Drug Public Opinion Monitoring Center of China Health Media Group-Participating in the Special Rectification of Food and Drug Rumors}

Five million WeChat public numbers and more than $5000 \mathrm{WeChat}$ public numbers of government and industry have joined the China Health Media Group Food and Drug Public opinion Monitoring Center, according to the official WeChat platform of the China Medical Journal.

China Health Media Group Food and Drug Public opinion Monitoring Center is specialized in food and drug public opinion monitoring and analysis services to provide early warning and disposal advice for the regulatory authorities. WeChat joined its food and drug monitoring system at the right time to facilitate the government's special treatment of food and drug rumors. WeChat, an online social platform, is more popular than official websites such as China's Food and Drug Administration. Actively cooperating with authoritative organizations, cooperating with the special rectification work of relevant departments, promoting the healthy development of the national food and drug industry. WeChat itself has also kept pace with the times.

\section{Conclusion and Prospect}

As a provider of network services, network operators have platform advantages for rumor management; As profitable network operating enterprises, they should bear the social responsibility to dispel rumors while benefiting; As a booster platform for spreading rumors, they have a legal obligation to deal with rumors. WeChat is a social platform with the largest number of users and the most active users in China. The rumor disinformation in food and drug rumor is worthy of consideration and reference by the major network operation media.

First of all, network operators should learn WeChat disinformation means, guide the public to participate, absorb social authority organizations, join the national professional public opinion regulatory agencies, and do more to refute rumors. After all, network rumor management is not overnight, need social participation. Network operators should also adapt to local conditions, according to the characteristics of their own network platform users and platform characteristics to open up suitable for their own disinformation means.

Secondly, the legal basis for the punishment of rumor-mongering is insufficient, and the process of pursuing responsibility is cumbersome. Network operators themselves should strengthen supervision and control of public opinion. At the same time, the state legislature should keep pace with the times, learn the advanced experience of foreign rumor management, perfect the law and make good use of the network platform to deal with the rumor.

Finally, the public opinion information has the inherent alienation gene5. The rumor production is inevitable, but the influence actually depends on the social public attitude and the behavior. Internet users should Make good use of the complaints and supervision functions of the network platform to maintain a healthy network environment instead of believing in ballads, spreading rumors.

If you follow the "checklist" your paper will conform to the requirements of the publisher and facilitate a problem-free publication process.

\section{References}

[1]. China Internet Network Information Center, Li Jing. The 41st report on the Development of the Internet in China [J]. China Radio, 2018 (3): 96-96.

[2]. Wang Fu-Zhong. The effect, function and significance of "interpretation" on the application of law in criminal cases such as libel by information network [J]. New Campus, 2014 (7): 39-39.

[3]. Shen Chao, Zhou Shu-Yi, Zhu Heng-Min. A study on the role of Group in the spread of Internet rumors [J]. Intelligence Magazine, 2016,35 (11): 113-118. 
[4]. A comprehensive publication. The passage of the Law of the people's Republic of China on Cybersecurity will take effect on June 1, 2017 [J]. Dual-Use Technology and products, 2016 (23): 4-4.

[5]. Li Hao-Qing. A study on the Evolution of Network derived Public opinion based on Information alienation Theory and its Countermeasures: taking the Governance of Internet rumors as an example [J]. Modern Intelligence, 2015, 35 (5): 4-8. 